\title{
New biomarkers and therapeutics can be discovered during COPD-lung cancer transition
}

\author{
Xiangdong Wang
}

Received: 29 June 2016 / Accepted: 30 June 2016 / Published online: 13 July 2016

(C) Springer Science+Business Media Dordrecht 2016

Chronic obstructive pulmonary disease (COPD) has been found to be associated with lung cancer regardless of a patient's smoking history. Of 11,888 incident cases of lung cancer, $23 \%$ had a prior diagnosis of COPD compared with only $6 \%$ of the 37,605 controls (Powell et al. 2013). The prevalence of COPD in lung cancer patients varies from 8 to $50 \%$, and the annual incidence of lung cancer arising from COPD is $0.8-1.2 \%$. This indicates that $\sim 1 \%$ of COPD patients develop lung cancer each year, while only $0.2 \%$ of patients with normal pulmonary function develop lung cancer. The Environment and Genetics in a Lung Cancer Etiology population-based case-control study showed that lung cancer risk was increased among individuals with chronic bronchitis, emphysema, and COPD (Sekine et al. 2012). COPD co-exists with $40-70 \%$ of lung cancer patients, and $50 \%$ of newly diagnosed lung cancer patients had COPD whereas only $8 \%$ of smokers without lung cancer had COPD. Smokers with COPD are more susceptible to lung cancer.

Epidemiological studies indicate that tobacco smoke exposure accounts for nearly $90 \%$ of cases of COPD and lung cancer. However, genetic factors may explain why $10-30 \%$ of smokers develop to COPD, even though genetic network and gene set enrichment in cigarette smoking were found to be correlated with the

X. Wang $(\bowtie)$

Clinical Science Institute of Fudan University Zhongshan Hospital, Shanghai Medical School, Shanghai, China e-mail: Xiangdong.wang@clintransmed.org development of lung cancer (Fang et al. 2013). COPD is closely linked to susceptibility to lung cancer and acts as the most important risk factor for lung cancer among smokers, predating lung cancer in up to $80 \%$ of cases. Genome-wide association studies on lung cancer, lung function, and COPD have identified a number of overlapping "susceptibility" loci. With stringent phenotyping, several overlapping loci are independently associated with both COPD and lung cancer, but with pulmonary inflammation and apoptotic processes mediated by the bronchial epithelium (Bondarczuk and PiotrowskaSeget 2013; Corbel et al. 2002; Bao et al. 2016a). Some lung cancer-specific genetic variants were identified as "COPD-related" genetic variants associated with clinical variables (Young et al. 2011).

Gene and protein expression profiles were initially investigated among patients with stable COPD, acute exacerbation of COPD (AECOPD), COPD with or without smoking, and COPD with or without lung cancer, to compare with those of healthy or health smokers (Chen et al. 2012). A number of disease-, stage-, duration-, or therapy-specific biomarkers were selected to screen susceptible populations of COPD or lung cancer. Using clinical bioinformatic techniques, the specificity, biological function, or prevalence of selected target genes or proteins from patients with AECOPD were furthermore validated in association with different types, survival rate, or potential mechanism of lung cancer (Ye et al. 2015). Chronic inflammation associated with COPD likely plays a role in the pathogenesis of lung cancer, and acute exacerbation can be the critical factor in the tumorigenesis of lung cancer. Inflammation 
in AECOPD may result in repeated airway epithelial injury, accompanying high cell turnover rates and propagation of DNA errors and resulting in amplification of the carcinogenic effects (Raviv et al. 2011).

It would be more value to screen and evaluate multidimensional gene profiles of leukocytes in patients with stable COPD, AECOPD, COPD with or without smoking, and COPD with or without lung cancer, to compare with those of healthy non-smokers or health smokers (Wu et al. 2014a, b). Multiple somatic aberrations of leukocytes should be identified, including copy number alterations and point mutations by measuring gene expression, sequencing, and epigenetics (Bao et al. 2016b). It is important to identify causal variants and emergent vulnerabilities of leukocytes that arise as a consequence of genetic alterations and may be the initiator for the tumorigenesis of lung cancer. Wholegenome small hairpin RNA "dropout screens" can be helpful to identify vulnerabilities in COPD and lung cancer, including candidate "drivers," and functional genomic properties of lung cancer cells or leukocytes in lung cancer, using a hierarchical linear regression algorithm to score our screen results and integrate them with accompanying detailed genetic and proteomic information (Marcotte et al. 2016). We need to provide solid evidence for precision medicine in lung cancer to compare gene essentiality with drug sensitivity data and investigate potential mechanisms of drug resistance, effects of anti-cancer drug combination, and potential therapeutic target (Wang 2016; He et al. 2015; Chen et al. 2015).

TGF- $\beta$ signaling has the duality of pro-tumorigenic or tumor suppressive roles in cancers associated with the frequent inactivation of the TGF- $\beta$ mediator Smad4. TGF- $\beta$ was found to induce an epithelialmesenchymal transition (EMT) in airway epithelial cells and considered as a pro-tumorigenic event in the tumorigenesis of lung cancer from COPD (Shi et al. 2016). EMT becomes lethal by converting TGF- $\beta$-induced SRY-box 4 (Sox4), phosphoinositide 3-kinase (PI3K), or osteopontin (OPN) from an enforcer of tumorigenesis into a promoter of apoptosis in TGF- $\beta$-sensitive lung cancer and COPD cells. TGF- $\beta$-induced EMT was accompanied with increased collagen type I deposition, through connective tissue growth factor (CTGF) production and PI3K signaling pathway. Treatment with PI3K inhibitors significantly attenuated the upregulation of TGF- $\beta$-stimulated CTGF expression, inhibited the process of EMT and collagen type I synthesis in lung epithelial cells. It is important to furthermore clarify the EMT-linked remodeling of the cellular transcription factor landscape in COPD and lung cancer. Smad4 is required for EMT but dispensable for Sox 4 induction by TGF- $\beta$ (David et al. 2016). TGF- $\beta$-induced Sox4, PI3K, or OPN may be geared to bolster progenitor identity, whereas simultaneous Smad4-, PI3K-, or OPN-dependent EMT strips Sox4 an essential partner in oncogenesis from COPD to lung cancer. More information regarding TGF- $\beta$ tumor suppression functions through the EMT-mediated disruption of a specific transcriptional network and identification of gene positioning factors using high-throughput imaging mapping should be possible.

It is more important to define the heterogeneity and homogeneity between genetically identical cells from COPD or lung cancer, or normal lung epithelial cells in the conditions of inflammatory stimulate (COPD) or tumorigenic agents (lung cancer). We recently established the technique of the single-cell sequencing that has the capacity of measuring the heterogeneity between single cells from different organs within a body or locations within a tumor (Wang 2015). The variability of cytoplasmic transcript abundance can be furthermore investigated using image-based transcriptomics in single human cells. Phenotypes and mechanisms of nuclear retention and transport of transcripts between the nucleus and the cytoplasm in stochastic transcriptional fluctuations should be defined in gene expression profiles of single cells from COPD or lung cancer using mathematical modeling and multivariate models of the phenotypic state and of single cells (Battich et al. 2015). The roles of TGF- $\beta$-induced Sox 4 , PI3K, or OPN pathways across genes in the complex regulatory system should be explored to control the observed variability of transcript abundance between individual cells in the tumorigenesis of lung cancer from COPD using computational multiplexing. It should be evident that the cellular compartmentalization is altered and chromosome repositioning occurs to confine transcriptional noise to the nucleus and prevent it from interfering with the control of single-cell transcript abundance in the cytoplasm in lung cancer from COPD.

In conclusion, chronic lung disease has become the leading cause of mortality and morbidity. It is critical and important to understand the interaction and network of driver molecules and develop new biomarkers and therapeutics during the transformation from chronic lung inflammation to cancer. Clinical studies clearly demonstrate 
the correlation between COPD and lung cancer and special attention to explore molecular mechanisms of COPDlung cancer transits by integrating gene sequencing and epigenetics, proteomic profiles, and metabolomics is called for. Furthermore, the cellular compartmentalization and chromosome repositioning as well as single-cell transcript abundance and biology function should be investigated in lung cancer arising from COPD.

\section{References}

Bao L Zhang Y, et al. Variations of chromosome 2 gene expressions among patients with lung cancer or non-cancer. Cell Biol Toxicol. 2016a. doi:10.1007/s10565-016-9343-Z.

Bao L, Diao H, Dong N, Su X, Wang B, Mo Q, Yu H, Wang XD, Chen $\mathrm{C}$. Histone deacetylase inhibitor induces cell apoptosis and cycle arrest in lung cancer cells via mitochondrial injury and p53 up-acetylation. Cell Biol Toxicol 2016b (in press).

Battich N, Stoeger T, Pelkmans L. Control of transcript variability in single mammalian cells. Cell. 2015;163(7):1596-610.

Bondarczuk K, Piotrowska-Seget Z. Molecular basis of active copper resistance mechanisms in Gram-negative bacteria. Cell Biol Toxicol. 2013;29(6):397-405.

Chen H, Song ZJ, Qian MJ, Bai CX, Wang XD. Selection of disease-specific biomarkers by integrating inflammatory mediators with clinical informatics in AECOPD patients: a preliminary study. J Cell Mol Med. 2012;16:1286-97.

Chen C, He M, Zhu Y, Shi L, Wang XD. Five critical elements to ensure the precision medicine. Cancer Metastasis Rev. 2015;34(2):313-8.

Corbel M, Belleguic C, Boichot E, Lagente V. Involvement of gelatinases (MMP-2 and MMP-9) in the development of airway inflammation and pulmonary fibrosis. Cell Biol Toxicol. 2002;18(1):51-61.

David CJ, Huang YH, Chen M, et al. TGF- $\beta$ tumor suppression through a lethal EMT. Cell. 2016;164(5):1015-30.
Fang X, Netzer M, Baumgartner C, Bai C, Wang XD. Genetic network and gene set enrichment analysis to identify biomarkers related to cigarette smoking and lung cancer. Cancer Treat Rev. 2013;39(1):77-88.

He M, Xia J, Shehab M, Wang XD. The development of precision medicine in clinical practice. Clin Transl Med. 2015;4:28.

Marcotte R, Sayad A, Brown KR, et al. Functional genomic landscape of human breast cancer drivers, vulnerabilities, and resistance. Cell. 2016;164(1-2):293-309.

Powell HA, Iyen-Omofoman B, Baldwin DR, Hubbard RB, Tata LJ. Chronic obstructive pulmonary disease and risk of lung cancer: the importance of smoking and timing of diagnosis. $\mathrm{J}$ Thorac Oncol. 2013;8(1):6-11.

Raviv S, Hawkins KA, DeCamp MM, Kalhan R. Lung cancer in chronic obstructive pulmonary disease. Am J Respir Crit Care Med. 2011;183(9):1138-46.

Sekine Y, Katsura H, Koh E, Hiroshima K, Fujisawa T. Early detection of COPD is important for lung cancer surveillance. Eur Respir J. 2012;39(5):1230-40.

Shi L, Dong N, Fang XC, Wang XD. Regulatory mechanisms of TGF- $\beta 1$-induced fibrogenesis of human alveolar epithelial cells. J Cell Mol Med 2016 (in press).

Wang XD. Single cell sequencing and systems immunology. Ed Wang, Published by Springer Publication. 2015.

Wang XD. Gene mutation-based and specific therapies in precision medicine. J Cell Mol Med. 2016;20(4):577-80.

Wu X, Sun X, Chen C, Bai C, Wang XD. Dynamic gene expressions of peripheral blood mononuclear cells in patients with acute exacerbation of chronic obstructive pulmonary disease: a preliminary study. Crit Care. 2014a;18(6):508.

Wu X, Yuan B, López E, Bai C, Wang XD. Gene polymorphisms and chronic obstructive pulmonary disease. J Cell Mol Med. 2014b;18(1):15-26.

Ye L, Wang J, Wang L, Hou J, Qian P, Wang XD. Roles of CEACAM1 in cell communication and signaling of lung cancer and other diseases. Cancer Metastasis Rev. 2015;34(2):347-57.

Young RP, Hopkins RJ, Gamble GD, Etzel C, El-Zein R, Crapo JD. Genetic evidence linking lung cancer and COPD: a new perspective. Appl Clin Genet. 2011;4:99-111. 